\title{
A SPECTRAL MUTISCALE HYBRIDIZABLE DISCONTINUOUS GALERKIN METHOD FOR SECOND ORDER ELLIPTIC PROBLEMS
}

\author{
YALCHIN EFENDIEV, RAYTCHO LAZAROV, MINAM MOON, AND KE SHI \\ Dedicated to Mary Wheeler on the occasion of her 75-th anniversary
}

\begin{abstract}
We design a multiscale model reduction framework within the hybridizable discontinuous Galerkin finite element method. Our approach uses local snapshot spaces and local spectral decomposition following the concept of Generalized Multiscale Finite Element Methods. We propose several multiscale finite element spaces on the coarse faces that provide a reduced dimensional approximation for numerical traces within the HDG framework. We provide a general framework for systematic construction of multiscale trace spaces. Using local snapshots, we avoid high dimensional representation of trace spaces and use some local features of the solution space in constructing a low dimensional trace space. We investigate the solvability and numerically study the performance of the proposed method on a representative number of numerical examples.
\end{abstract}

\section{INTRODUCTION}

In this paper, we propose a novel hybridized discontinuous Galerkin multiscale finite element method for flows in highly heterogeneous porous media. We consider the second-order elliptic differential equation

$$
-\nabla \cdot(\kappa(x) \nabla u)=f(x) \text { in } \Omega,
$$

where $\Omega$ is a bounded polyhedral domain in $\mathbb{R}^{n}, n=2,3$. We assume that $f \in L^{2}(\Omega)$ and the coefficient $\kappa(x) \geq \kappa_{0}>0$ represents the permeability of a highly heterogeneous porous media with multiple scales and high contrast (the ratio between the maximum and minimum values of $\kappa$ ). Such problems appear in many industrial, scientific, and environmental applications. Resolving all scales requires very fine meshes and this makes the corresponding algebraic problem (arising form the finite element, finite volume, or mixed FEM approximations) a challenging and often prohibitively expensive task. This necessitates the use of reduced-order methods or specialized techniques for solving the algebraic system, e.g. multigrid (e.g., [7, 15]), multilevel methods (e.g., [31, 32]), and/or domain decomposition techniques (e.g., [29, 18, 22, $23,25,30])$.

More recently, new classes of reduced-order methods have been introduced and used in various applications. These include Galerkin multiscale finite element method (e.g., [3, 11, 14, 19, 20, 21]), mixed multiscale finite element methods (e.g., $[1,2,5,27]$ ), the multiscale finite volume method (see, e.g., [28]), mortar multiscale methods (see e.g., [8, 33]), and variational multiscale methods (see e.g., [26]). In this paper, we use the Generalized Multiscale Finite

Date: Started October 2013, today is August 4, 2014.

Key words and phrases. Multiscale finite element method, hybridizable discontinuous Galerkin, spectral basis function.

1

(C) 2015. This manuscript version is made available under the Elsevier user license

http://www.elsevier.com/open-access/userlicense/1.0/ 
Element [16] concept to construct a local reduced-order approximation for the solution space. In particular, the local snapshot functions and spectral decomposition of the snapshot space are constructed to approximate the solution in each coarse patch. The multiscale approach allows bringing the local properties of the solution into a global coarse-grid problem in a systematic way that substantially reduces the number of coupled degrees of freedom in the algebraic system. The local snapshot solutions are computed separately (possibly in parallel or off-line) on each coarse block. In general, these solutions are discontinuous along the coarse grid interfaces and, consequently, robust discontinuous Galerkin approaches are needed.

One of the earlier efficient mixed finite element methods on multi-block grids were devised by Mary Wheeler and her co-authors in [6] by using the framework of domain decomposition method and mortaring technique. Mortaring technique (see, e.g. [10]) was introduced to accommodate approximations on subdomains that could be meshed independently and use non-conforming finite element spaces. This technique introduces an auxiliary space on each subdomain boundary associated with the Lagrange multiplier that imposes the continuity constraint on the global approximation. The classical mortaring, devised for the needs of domain decomposition methods, [10], has been adapted recently as multiscale discretization by Mary Wheeler and her collaborators (e.g. $[8,9]$ ). In a two-scale (two-grid, fine and coarse) method, the aim is to resolve the local heterogeneities on the fine grid introduced on each coarse block and then glue these approximations together via mortar spaces. The functions in these spaces, which play the role of Lagrange multipliers, are defined on the boundaries of the coarse partition. In order to design a stable method, the mortar spaces have to satisfy a proper inf-sup condition. This approach is shown to be well suited for problems with heterogeneous media and a number of efficient methods and implementations have been proposed, studied, and used for solving a variety of applied problems (e.g. [3, 4, 8]).

In this paper, we study multiscale model reduction techniques within the framework of the Hybridizable Discontinuous Galerkin method (HDG). The hybridization of the finite element methods, as outlined in [12], provides ample possibilities for "gluing" together various finite element approximations. The mechanism of this "glue" is based on the notions of numerical trace and numerical flux. Numerical trace is a single valued function on the finite element interfaces and belongs to a certain Lagrange multiplier space which is used to solve the global problem. The well-posedness and accuracy are ensured by a proper choice of the numerical flux, that involves a stabilization parameter $\tau$ (see, e.g. [12]). Standard approaches for selecting numerical traces involve the use of piecewise polynomials. However, the solution along the boundaries of a coarse grid can not have an arbitrary form and can be represented by a much lower dimensional space. In this paper, we seek a low dimensional representation of trace spaces. Our approach relies on local basis functions that are constructed independently (and in an offline stage) on each coarse-grid cell. The coupling of these local functions is provided by the HDG technology, where the role of the "glue" plays the space of the numerical trace whose construction is the main goal of this paper. In this paper, we construct several multiscale spaces for the numerical trace that provide reasonable approximations of the solution and results in a stable multi-scale method. The efficiency of the proposed methods is demonstrated on a set of numerical experiments with flows in high-contrast media.

The paper is organized in the following way. In Section 2, we present our model problem by using two grids, fine (that resolves all scales of the heterogeneity in $\kappa$ ) and coarse (where the solution will be sought) and hybridizable discontinuous Galerkin method. In Section 3, we 


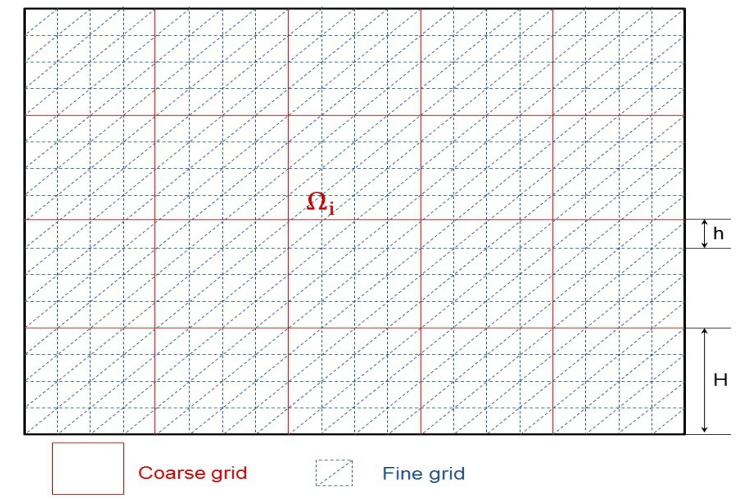

FiguRE 1. Fine and coarse grids: the coarse-grid blocks are squares while the fine grid finite elements are triangles.

describe the multiscale FEM based on HDG framework and present several different choices of spectral basis functions. In Section 4, we present some numerical experiments and report the error of HDG with the constructed spectral basis spaces and in Section 5, we discuss the numerical results.

\section{Preliminary}

2.1. Fine and coarse grids. The mutiscale finite element method for the equation (1) is based on the hybridizable discontinuous Galerkin method (HDG) and uses the concept of two-grid approximation.

We split the domain $\Omega$ into disjoint polygonal subdomains $\left\{\Omega_{i}\right\}_{i=1}^{N}$ of diameter $O\left(H_{i}\right)$ so that $\bar{\Omega}=\cup_{i=1}^{N} \bar{\Omega}_{i}$, see e.g. Figure 1 . We denote by $F_{H}$ an face (edge) of the subdomain $\Omega_{i}$, if $F_{H}$ is either shared by $\Omega_{i}$ and its neighboring subdomain $\Omega_{j}$, i.e. $F_{H}=\bar{\Omega}_{i} \cap \bar{\Omega}_{j}$ or $F_{H}=\bar{\Omega}_{i} \cap \partial \Omega$. Then $\mathcal{E}_{H}\left(\Omega_{i}\right)$ denotes the set of all coarse edges of a subdomain $\Omega_{i}$ and $\mathcal{E}_{H}=\cup_{i=1}^{N} \mathcal{E}_{H}\left(\Omega_{i}\right)$.

In each subdomain $\Omega_{i}$ we introduce a shape regular triangulation $\mathcal{T}_{h}\left(\Omega_{i}\right)$ with triangular elements and maximum mesh-size $h_{i}$. Faces (edges) of this triangulation are denoted by $F_{h}$. Let $\mathcal{T}_{h}=\cup_{i=1}^{N} \mathcal{T}_{h}\left(\Omega_{i}\right), \mathcal{E}_{h}\left(\Omega_{i}\right)$ be the set of all faces of the triangulation $\mathcal{T}_{h}\left(\Omega_{i}\right)$ and $\mathcal{E}_{h}^{0}\left(\Omega_{i}\right)$ be the set of all interior edges of the triangulation $\mathcal{T}_{h}\left(\Omega_{i}\right)$ and set $\mathcal{E}_{h}=\cup_{i=1}^{N} \mathcal{E}_{h}\left(\Omega_{i}\right)$. Further $\mathbf{x}_{i}$, $i=1, \ldots, N_{v}$, denote the nodes in the partition $\mathcal{T}_{h}$. Note that the scale $H_{i}$ is associated only with the subdomains $\Omega_{i}$. Similar construction is assumed in $3-\mathrm{D}$ with $\Omega_{i}$ being polyhedra while the fine-grid elements are simplices or hexahedra.

2.2. Fine-scale hybridizable discontinuous Galerkin method. In this subsection, we present HDG method for the problem (1) on the fine-grid $\mathcal{T}_{h}$. We first write the equation (1) with homogeneous Dirichlet boundary conditions in a mixed form:

$$
\begin{aligned}
& \alpha \boldsymbol{q}+\nabla u=0 \text { in } \Omega \text {, } \\
& \nabla \cdot \boldsymbol{q}=f \text { in } \Omega, \\
& u=0 \text { on } \partial \Omega
\end{aligned}
$$


with $\alpha(x)=\kappa(x)^{-1}$ and further introduce the finite element spaces that are used in the HDG method:

$$
\begin{aligned}
W_{h} & :=\left\{w \in L^{2}\left(\mathcal{T}_{h}\right):\left.w\right|_{T} \in W(T), T \in \mathcal{T}_{h}\right\}, \\
\boldsymbol{V}_{h} & :=\left\{\boldsymbol{r} \in \boldsymbol{L}^{2}\left(\mathcal{T}_{h}\right):\left.\boldsymbol{r}\right|_{T} \in \boldsymbol{V}(T), T \in \mathcal{T}_{h}\right\}, \\
M_{h} & :=\left\{\mu \in L^{2}\left(\mathcal{E}_{h}\right):\left.\mu\right|_{F_{h}} \in M\left(F_{h}\right), F_{h} \in \mathcal{E}_{h},\left.\mu\right|_{\partial \Omega}=0\right\}, \\
M_{h}^{0} & :=\left\{\mu \in L^{2}\left(\mathcal{E}_{h}\right):\left.\mu\right|_{F_{h}} \in M\left(F_{h}\right), F_{h} \in \mathcal{E}_{h},\left.\mu\right|_{\partial T}=0\right\}, \\
M_{H} & :=\left\{\mu \in L^{2}\left(\mathcal{E}_{H}\right):\left.\mu\right|_{F_{H}} \in M\left(F_{H}\right), F_{H} \in \mathcal{E}_{H},\left.\mu\right|_{\partial \Omega}=0\right\}, \\
M_{h, H} & :=M_{h}^{0} \oplus M_{H} .
\end{aligned}
$$

Here for $T \in \mathcal{T}_{h}$ the spaces $W(T), \boldsymbol{V}(T)$, and $M\left(F_{h}\right)$ are suitable finite element spaces (see, e.g. [12]). For example, all three space can consist of linear functions. The focus in this paper will be the choice of the space $M_{H}$.

Further, we assume that the specified above spaces have "nodal" bases so that the coefficients in the representation of a function with respect to the nodal bases form column vectors in an Euclidean space which dimension is equal to the dimension of the finite elements space. Often we shall use the same notation for the function and for its vector representation (with respect to the nodal bases) when this does not lead to a confusion.

The multiscale HDG method is: Find $\left(u_{h}, \boldsymbol{q}_{h}, \widehat{u}_{h, H}\right) \in W_{h} \times \boldsymbol{V}_{h} \times M_{h, H}$ such that

$$
\begin{array}{rlrlrl}
\left(\alpha \boldsymbol{q}_{h}, \boldsymbol{r}\right)_{\mathcal{T}_{h}}-\left(u_{h}, \nabla \cdot \boldsymbol{r}\right)_{\mathcal{T}_{h}}+\left\langle\widehat{u}_{h, H}, \boldsymbol{r} \cdot \boldsymbol{n}\right\rangle_{\partial \mathcal{T}_{h}} & =0 & & \forall \boldsymbol{r} \in \boldsymbol{V}_{h}, \\
-\left(\boldsymbol{q}_{h}, \nabla w\right)_{\mathcal{T}_{h}} & +\left\langle\widehat{\boldsymbol{q}}_{h, H} \cdot \boldsymbol{n}, w\right\rangle_{\partial \mathcal{T}_{h}} & =(f, w)_{\mathcal{T}_{h}} & & \forall w \in W_{h}, \\
& \left\langle\widehat{\boldsymbol{q}}_{h, H} \cdot \boldsymbol{n}, \mu\right\rangle_{\partial \mathcal{T}_{h}} & =0 & & \forall \mu \in M_{h, H}, \\
& \widehat{u}_{h, H} & & & & \text { on } \partial \Omega .
\end{array}
$$

Here, $\widehat{u}_{h, H}$ and $\widehat{\boldsymbol{q}}_{h, H}$ are the numerical trace and the numerical flux, correspondingly. We write $(\eta, \zeta)_{\mathcal{T}_{h}}:=\sum_{T \in \mathcal{T}_{h}}(\eta, \zeta)_{T}$, where $(\eta, \zeta)_{D}$ denotes the integral of $\eta \zeta$ over the domain $D \in \mathbb{R}^{n}$ and $\langle\eta, \zeta\rangle_{\partial \mathcal{T}_{h}}:=\sum_{T \in \mathcal{T}_{h}}\langle\eta, \zeta\rangle_{\partial T}$, where $\langle\eta, \zeta\rangle_{\partial D}$ denotes the integral of $\eta \zeta$ over the boundary of the domain $\partial D \subset \mathbb{R}^{n-1}$. Consequently, $\langle\eta, \zeta\rangle_{\partial \Omega_{i}}:=\int_{\partial \Omega_{i}} \eta \zeta d s$. The HDG method formulation is completed with the definition of the normal component of the numerical trace :

$$
\widehat{\boldsymbol{q}}_{h, H} \cdot \boldsymbol{n}=\boldsymbol{q}_{h} \cdot \boldsymbol{n}+\tau\left(u_{h}-\widehat{u}_{h, H}\right),
$$

where $\tau$ is a non-negative stabilization parameter. We remark that if there is only one coarse grid (i.e. $\Omega_{i}=\Omega, i=1$ ) then this is the standard HDG method [12].

\section{The mutiscale hybridizable discontinuous Galerkin method}

In this section, we will construct local mutiscale basis functions for $M_{H}$. For this, we propose the structure of mutiscale HDG method and local spectral problems are designed and solved to compute multiscale basis functions. To keep our presentation simple, we first show the structure of the mutiscale HDG with given multiscale functions. Next, we will discuss how these functions can be generated.

3.1. The mutiscale HDG framework. The main feature of this method is that it could be implemented in such a way that we need to solve global system on the coarse mesh only. To do this, we split the third equation of (4) into two equations by testing separately with $\mu \in M_{h}^{0}$ and $\mu \in M_{H}$ so that

$$
\left\langle\widehat{\boldsymbol{q}}_{h, H} \cdot \boldsymbol{n}, \mu\right\rangle_{\partial \mathcal{T}_{h}}=0 \quad \forall \mu \in M_{h}^{0}
$$


and

$$
\left\langle\widehat{\boldsymbol{q}}_{h, H} \cdot \boldsymbol{n}, \mu\right\rangle_{\partial \Omega_{i}}=0 \quad \forall \mu \in M_{H} \text { and } i=1, \ldots, N .
$$

Now because of the structure of the space $M_{h}^{0}$ we can implement the solution of equation (6) independently on each subdomain $\Omega_{i}$. Indeed, for a particular subdomain $\Omega_{i}$, let $\widehat{u}_{h, H}=\xi_{H}$, where $\xi_{H} \in M_{H}$. Then on $\Omega_{i}$, we can find the solution $\left.\left(u_{h}, \widehat{\boldsymbol{q}}_{h}, \widehat{u}_{h, H}\right)\right|_{\Omega_{i}}$ by restricting the equation (4) to $\Omega_{i}$ :

$$
\begin{aligned}
& \left(\alpha \boldsymbol{q}_{h}, \boldsymbol{r}\right)_{\mathcal{T}_{h}\left(\Omega_{i}\right)}-\left(u_{h}, \nabla \cdot \boldsymbol{r}\right)_{\mathcal{T}_{h}\left(\Omega_{i}\right)}+\left\langle\widehat{u}_{h, H}, \boldsymbol{r} \cdot \boldsymbol{n}\right\rangle_{\partial \mathcal{T}_{h}\left(\Omega_{i}\right)}=0, \\
& -\left(\boldsymbol{q}_{h}, \nabla w\right)_{\mathcal{T}_{h}\left(\Omega_{i}\right)}+\left\langle\widehat{\boldsymbol{q}}_{h, H} \cdot \boldsymbol{n}, w\right\rangle_{\partial \mathcal{T}_{h}\left(\Omega_{i}\right)}=(f, w)_{\mathcal{T}_{h}\left(\Omega_{i}\right)}, \\
& \left\langle\widehat{\boldsymbol{q}}_{h, H} \cdot \boldsymbol{n}, \mu\right\rangle_{\partial \mathcal{T}_{h}\left(\Omega_{i}\right)}=0, \\
& \widehat{u}_{h, H} \quad=\xi_{H} \text { on } \partial \Omega_{i}
\end{aligned}
$$

for all $\left.(w, \boldsymbol{r}, \mu) \in W_{h}\right|_{\Omega_{i}} \times\left.\boldsymbol{V}_{h}\right|_{\Omega_{i}} \times\left. M_{h}^{0}\right|_{\mathcal{E}_{h}^{0}\left(\Omega_{i}\right)}$. Here, we have used the relation (5) for the "numerical flux" $\widehat{\boldsymbol{q}}_{h, H}$. In fact, the above system is the regular HDG methods defined on $\Omega_{i}$. From [12], we already know that under proper choice of the spaces $W(T), \boldsymbol{V}(T)$, and $M\left(F_{h}\right)$ this problem is stable. Using superposition principle the solution of equation (8) can be further split into two parts, namely,

$$
\left(\boldsymbol{q}_{h}, u_{h}, \widehat{u}_{h, H}\right)=\left(\boldsymbol{q}_{h}(f), u_{h}(f), \widehat{u}_{h, H}(f)\right)+\left(\boldsymbol{q}_{h}\left(\xi_{H}\right), u_{h}\left(\xi_{H}\right), \widehat{u}_{h, H}\left(\xi_{H}\right)\right),
$$

where $\left(\boldsymbol{q}_{h}(f), u_{h}(f), \hat{u}_{h, H}(f)\right)$ satisfies

$$
\begin{aligned}
& \left(\alpha \boldsymbol{q}_{h}(f), \boldsymbol{r}\right)_{\mathcal{T}_{h}\left(\Omega_{i}\right)}-\left(u_{h}(f), \nabla \cdot \boldsymbol{r}\right)_{\mathcal{T}_{h}\left(\Omega_{i}\right)}+\left\langle\widehat{u}_{h, H}(f), \boldsymbol{r} \cdot \boldsymbol{n}\right\rangle_{\partial \mathcal{T}_{h}\left(\Omega_{i}\right)}=0, \\
& -\left(\boldsymbol{q}_{h}(f), \nabla w\right)_{\mathcal{T}_{h}\left(\Omega_{i}\right)}+\left\langle\widehat{\boldsymbol{q}}_{h, H}(f) \cdot \boldsymbol{n}, w\right\rangle_{\partial \mathcal{T}_{h}\left(\Omega_{i}\right)}=(f, w)_{\mathcal{T}_{h}\left(\Omega_{i}\right)}, \\
& \left\langle\widehat{\boldsymbol{q}}_{h, H}(f) \cdot \boldsymbol{n}, \mu\right\rangle_{\partial \mathcal{T}_{h}\left(\Omega_{i}\right)}=0, \\
& \widehat{u}_{h, H}(f) \quad=0 \text { on } \partial \Omega_{i},
\end{aligned}
$$

for all $\left.(w, \boldsymbol{r}, \mu) \in W_{h}\right|_{\Omega_{i}} \times\left.\boldsymbol{V}_{h}\right|_{\Omega_{i}} \times\left. M_{h}^{0}\right|_{\mathcal{E}_{h}^{0}\left(\Omega_{i}\right)}$ and $\left(\boldsymbol{q}_{h}\left(\xi_{H}\right), u_{h}\left(\xi_{H}\right), \widehat{u}_{h, H}\left(\xi_{H}\right)\right)$ satisfies

$$
\begin{aligned}
& \left(\alpha \boldsymbol{q}_{h}\left(\xi_{H}\right), \boldsymbol{r}\right)_{\mathcal{T}_{h}\left(\Omega_{i}\right)}-\left(u_{h}\left(\xi_{H}\right), \nabla \cdot \boldsymbol{r}\right)_{\mathcal{T}_{h}\left(\Omega_{i}\right)}+\left\langle\widehat{u}_{h, H}\left(\xi_{H}\right), \boldsymbol{r} \cdot \boldsymbol{n}\right\rangle_{\partial \mathcal{T}_{h}\left(\Omega_{i}\right)}=0, \\
& -\left(\boldsymbol{q}_{h}\left(\xi_{H}\right), \nabla w\right)_{\mathcal{T}_{h}\left(\Omega_{i}\right)}+\left\langle\widehat{\boldsymbol{q}}_{h, H}\left(\xi_{H}\right) \cdot \boldsymbol{n}, w\right\rangle_{\partial \mathcal{T}_{h}\left(\Omega_{i}\right)}=0, \\
& \left\langle\widehat{\boldsymbol{q}}_{h, H}\left(\xi_{H}\right) \cdot \boldsymbol{n}, \mu\right\rangle_{\partial \mathcal{T}_{h}\left(\Omega_{i}\right)}=0, \\
& \widehat{u}_{h, H}\left(\xi_{H}\right) \quad=\xi_{H} \text { on } \partial \Omega_{i} \text {, }
\end{aligned}
$$

for all $\left.(w, \boldsymbol{r}, \mu) \in W_{h}\right|_{\Omega_{i}} \times\left.\boldsymbol{V}_{h}\right|_{\Omega_{i}} \times\left. M_{h}^{0}\right|_{\mathcal{E}_{h}^{0}\left(\Omega_{i}\right)}$.

Then the equation (7) reduces to finding $\xi_{H} \in M_{H}$ such that

$$
a\left(\xi_{H}, \mu\right)=l(\mu) \text { for all } \mu \in M_{H},
$$

where the bilinear form $a\left(\xi_{H}, \mu\right): M_{H} \times M_{H} \rightarrow \mathbb{R}$ and the linear form $l(\mu): M_{H} \rightarrow \mathbb{R}$ are defined as

$$
a\left(\xi_{H}, \mu\right):=\sum_{i=1}^{N}\left\langle\widehat{\boldsymbol{q}}_{h, H}\left(\xi_{H}\right), \mu\right\rangle_{\partial \Omega_{i}} \text { and } l(\mu):=-\sum_{i=1}^{N}\left\langle\widehat{\boldsymbol{q}}_{h, H}(f), \mu\right\rangle_{\partial \Omega_{i}} .
$$

We note that the same procedure can be applied for the case of non-homogeneous boundary data $u=g$ on $\partial \Omega$ as well.

To motivate the need for multiscale spaces for $M_{H}$, we first present numerical examples using polynomial functions for $M_{H}$. In Figure 2, the high-contrast coefficient $\kappa(x)$, fine-scale 


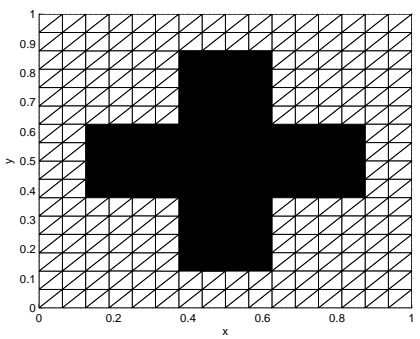

(A) Heterogeneous coefficient

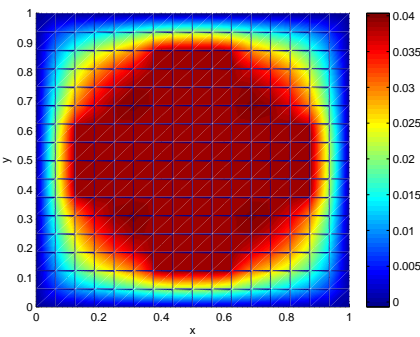

(в) Fine-scale solution

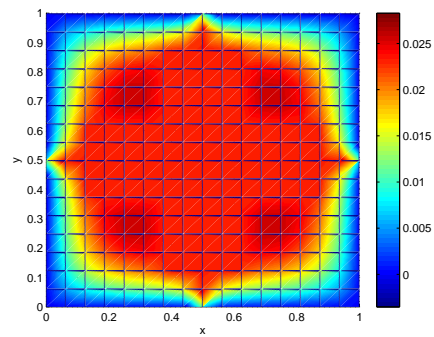

(C) Coarse-scale solution

FIgURE 2. Numerical results for mutiscale HDG framework with $M_{H}(F)=$ $\mathcal{P}_{2}(F), h=1 / 32, H=1 / 2$, and $\eta=10^{4}$.

solution, and multiscale solution with polynomial $M_{H}$ space are shown. In Figure 2-(A), the black region indicates the coefficient $\kappa(x)=\eta$ and the white region the coefficient $\kappa(x)=1$. The error between the fine-scale solution and the multiscale solution with polynomial space $M_{H}$ is $37.4 \%$. This representative example shows a need to go beyond piece-wise polynomial functions for $M_{H}$. In particular, when using spectral basis introduced in next sections, one can reduce the error below $5 \%$. Next, we will discuss the construction of new multiscale spaces.

3.2. A framework for constructing the multiscale space $M_{H}$. Although the multiscale space $M_{H}$ lives only on the coarse skeleton $\mathcal{E}_{H}$, it needs to involve some information of the permeability field within coarse blocks. A reasonable approach is to construct a number of snapshots using local solutions defined on a subdomain around a face $F$ (or set of faces) and then take the traces of those functions on $\mathcal{E}_{H}$ to form the space $M_{H}$. This will resemble traces of the global solution and avoid a very high-dimensional representation of the traces. In what follows, we present a general strategy of the construction of $M_{H}$ :

Step 1: Partition of skeleton. First, we decompose the skeleton $\mathcal{E}_{H}$ by defining a covering $\mathcal{E}_{H}=\cup \mathcal{E}_{i}$, where the summation is over all partitions $\mathcal{E}_{i}$. Here each $\mathcal{E}_{i}$ can be a single coarse edge or a union of several coarse edges. We allow overlaps between $\mathcal{E}_{i}, \mathcal{E}_{j}$, for some $i$ and $j$, $\mathcal{E}_{i} \cap \mathcal{E}_{j} \neq 0$. For each $\mathcal{E}_{i}$, we associate a support domain $\omega_{i}$ such that $\mathcal{E}_{i} \subset \bar{\omega}_{i}$. For instance, if $\mathcal{E}_{i}$ is a single coarse face then $\omega_{i}$ can be any one of the neighboring coarse blocks sharing this face or the union of the neighboring blocks that share the face.

Step 2: Local snapshot spaces $V^{\text {snap }}\left(\omega_{i}\right)$. For each $\omega_{i}$, we introduce the local snapshots $\left\{\phi_{j}\right\}_{j=1}^{N_{i}}$ that are defined on $\bar{\omega}_{i}$ and take

$$
V^{\text {snap }}\left(\omega_{i}\right)=\operatorname{span}\left\{\phi_{j}\right\}_{j=1}^{N_{i}} \text {. }
$$

The snapshots can be given explicitly or computed via solutions of local boundary value or local spectral problems in $\omega_{i}$. The snapshots can represent the fine-scale features of the solution and thus the choice of the snapshot space impacts the dimension of the local spectral problems.

Now, we present two examples for computing the snapshot space. These examples will be used in later sections.

The simplest choice would be a snapshot space $V^{\operatorname{snap}}\left(\omega_{i}\right)$ that consists of all fine-grid basis functions (see Section 3.3.1) defined on $\omega_{i}$. Such choice substantially reduces the computational cost associated with calculating the snapshot vectors. 
A second approach is based on local solutions of the problem. Let $\left\{\boldsymbol{x}_{l}\right\}_{l=1}^{N_{i}}$ be the set of all fine nodes on $\partial \omega_{i}$. For each $\omega_{i}$ we define the function $\phi_{l}$ as the finite element solution of the following local problem (in a discrete setting):

$$
\nabla \cdot\left(\kappa \nabla \phi_{l}\right)=0 \quad \text { in } \omega_{i}, \quad \phi_{l}=\delta_{l} \quad \text { on } \partial \omega_{i} .
$$

Here $\delta_{l}$ is a continuous piecewise linear function defined on $\partial \omega_{i}$ such that $\delta_{l}(x)=1$ on $\boldsymbol{x}_{l}$ and vanishes on all other nodes.

Step 3: Form the multiscale space $M_{H}$. For each $\omega_{i}$, we perform a spectral decomposition of $V^{\operatorname{snap}}\left(\omega_{i}\right)$ via some generalized eigenvalue problem

$$
\boldsymbol{A} \psi=\lambda \boldsymbol{M} \psi \text {. }
$$

Choices of the (stiffness) matrix $\boldsymbol{A}$ and the (mass) matrix $\boldsymbol{M}$ will be discussed in the following section. If the snapshot space consists of all fine-grid basis functions in $\omega_{i}$, the eigenvalue problem (14) reduces to solving the spectral problem with local stiffness and mass matrices defined on $\omega_{i}$. We order the eigenvalues as $0 \leq \lambda_{i, 1} \leq \lambda_{i, 2} \leq \cdots \leq \lambda_{i, N_{i}}$ and select the first $L_{i}$ eigenfunctions $\psi_{i, 1}, \ldots, \psi_{i, L_{i}}$ to form a smaller subspace $V^{\text {off }}\left(\omega_{i}\right)$ :

$$
V^{\text {off }}\left(\omega_{i}\right)=\operatorname{span}\left\{\psi_{i, j}\right\}_{j=1}^{L_{i}} \text {. }
$$

For each $\omega_{i}$, we form the local multiscale space $M_{H}(F)$ by taking the traces of the functions in $V^{\text {off }}$ on each $F$ and form the set of trace of $\psi_{i, j}$ on the face $F$ :

$$
\bigcup_{F \in \mathcal{E}_{i}}\left\{\left.\psi_{i, j}\right|_{F}: \psi_{i, j} \in V^{\mathrm{off}}\left(\omega_{i}\right), j=1, \ldots, L_{i}\right\} .
$$

Note that, in general, these functions will not be linearly independent so we need to perform a Proper Orthogonal Decomposition (POD) in order to eliminate the linearly dependent modes (e.g., [24]) or to select most important modes in order to further reduce the dimension to $L_{F}$. In this way, we produce the space $M_{H}(F)$. Finally, the multiscale space $M_{H}$ is defined as:

$$
M_{H}:=\bigcup_{F \in \mathcal{E}_{H}} M_{H}(F)
$$

Remark 3.1. Notice that in general $M_{H}$ is nonconforming in the sense that the functions in $M_{H}$ will have discontinuities on the coarse grid nodes. If it is desired to have a conforming space, in Step 1, we need to ensure that $\left\{\omega_{i}\right\}_{i=1}^{N}$ is a covering of the domain $\Omega$. Let $\left\{\chi_{i}\right\}_{i=1}^{N}$ be a set of partition of unity functions associated with the covering $\left\{\omega_{i}\right\}_{i=1}^{N}$. In Step 3, we modify the definition of $V^{\text {off }}$ by multiplying the space with the partition of unity function $\chi_{i}$ (so the functions are globally continuous and hence $M_{H}$ is conforming) to get

$$
V^{o f f}\left(\omega_{i}\right)=\operatorname{span}\left\{\chi_{i} \psi_{i, j}\right\}_{j=1}^{L_{i}} .
$$

3.3. Examples of multiscale spaces $M_{H}$. In this section, we present examples of the construction of multiscale spaces. The Mutiscale Spaces I (see Section 3.3.1 and 3.3.2) will use the traces of the multiscale functions constructed locally on each coarse block, where these multiscale functions are a low dimensional representation of the solution in each $\Omega_{i}$ and thus they provide a low dimensional trace space $M_{H}$. Furthermore, we remove linearly dependent components.

For the Mutiscale Spaces II, defined in Section 3.3.3 and 3.3.4, we employ local eigenvalue problem defined on coarse blocks that strictly contain the face $F$. Compared to the first 
approach, the second approach uses oversampling technique in computing $M_{H}$. The method proposed in Section 3.3.4 uses a partition of unity and provides a conforming $M_{H}$.

Due to the stabilization parameter $\tau$, the solvability of the system is independent of the choice of the multiscale space $M_{H}$. A brief discussion on this issue is in Section 3.4.

3.3.1. The Mutiscale Space I-a. For this choice, $\mathcal{E}_{i}$ refers to the boundary of a coarse-grid element $\Omega_{i}$ and $\omega_{i}=\Omega_{i}$ (see Figure 3 ). The snapshot space consists of all fine-grid functions in $\Omega_{i}$.

We generate the $V^{\text {off }}\left(\omega_{i}\right)$ by solving the local spectral problem described below. Let $X_{h}\left(\Omega_{i}\right)$ be the conforming finite element space of continuous piece-wise linear functions in $\mathcal{T}_{h}\left(\Omega_{i}\right)$. For any given subdomain $\Omega_{i}$, we find $\lambda$ and $\psi \in X_{h}\left(\Omega_{i}\right)$ such that

$$
\int_{\Omega_{i}} \kappa(x) \nabla \psi \nabla z d x=\lambda \int_{\Omega_{i}} \widetilde{\kappa}(x) \psi z d x \forall z \in X_{h}\left(\Omega_{i}\right) .
$$

$\widetilde{\kappa}$ is a properly selected weight. For a scalar permeability, we select $\widetilde{\kappa}=\kappa$. For tensor permeability, for the choice of $\widetilde{\kappa}$, we refer to [18]. We order the eigenvalues so that $0=\lambda_{i, 1} \leq$ $\lambda_{i, 2} \leq \cdots \leq \lambda_{i, N_{i}}$, where $N_{i}$ is the number of vertices of $\mathcal{T}_{h}\left(\Omega_{i}\right)$. Then $V^{\text {off }}\left(\Omega_{i}\right)$ is the space spanned by the eigenfunctions $\psi_{i, l}, l=1, \cdots, L_{i}$ corresponding to the first $L_{i}$ eigenvalues.

As we see from (3), the space $M_{H}$ is defined face-wise. Therefore, for a face $F \in \mathcal{E}_{H}$ common for the coarse blocks $\Omega_{i}$ and $\Omega_{j}$ we collect all traces of the constructed above eigenfunctions, namely

$$
F \in \mathcal{E}_{H}\left(\Omega_{i}\right) \cap \mathcal{E}_{H}\left(\Omega_{j}\right): \quad \psi_{i, l, F}=\left.\psi_{i, l}\right|_{F}, l=1, \cdots, L_{i}, \quad \psi_{j, l, F}=\left.\psi_{j, l}\right|_{F}, l=1, \cdots, L_{j} .
$$

The selected traces are in general linearly dependent, so we need to eliminate the linearly dependent ones. We use POD method and select the first $L_{F}$ linearly independent functions denoted by $\psi_{j, F}, j=1, \ldots, L_{F}$. Thus, for any $F \in \mathcal{E}_{H}$ we define

$$
M_{H}^{I_{a}}(F):=\operatorname{span}\left\{\psi_{j, F}, 0 \leq j \leq L_{F}\right\}, \quad \text { and } \quad M_{H}^{I_{a}}:=\bigcup_{F \in \mathcal{E}_{H}} M_{H}^{I_{a}}(F) .
$$

3.3.2. The Mutiscale Space $I-b$. The definition of $\omega_{i}$ and $\mathcal{E}_{i}$ are the same as in the case of the Multiscale Space I-a. Let $m_{\mathcal{E}_{i}}$ be the number of nodal basis function corresponding to the finegrid nodes on $\mathcal{E}_{i}$. Then the snapshot space is defined by the finite element approximations (we keep the same notation for discrete solution as the continuous solution in $(13)) \phi_{l}, l=1, \ldots, m_{\mathcal{E}_{i}}$ of the problem (13) on $X_{h}\left(\omega_{i}\right)$ :

$$
V^{\text {snap }}\left(\Omega_{i}\right)=\operatorname{span}\left\{\phi_{l}, 1 \leq l \leq m_{\mathcal{E}_{i}}\right\} .
$$

To compute local spectral problem, we define the matrices

$$
A_{i}^{\text {snap }}=\left[\int_{\Omega_{i}} \kappa \nabla \phi_{l} \nabla \phi_{l^{\prime}} d x\right]_{l, l^{\prime}=1,}^{m_{\mathcal{E}_{i}}}, M_{i}^{\text {snap }}=\left[\frac{1}{H} \int_{\mathcal{E}_{i}} \kappa \phi_{l} \phi_{l^{\prime}} d s\right]_{l, l^{\prime}=1}^{m_{\mathcal{E}_{i}}} .
$$

Using these matrices, we solve the following algebraic eigenvalue problem:

$$
A_{i}^{\text {snap }} \boldsymbol{\Sigma}_{i}=\Lambda_{i} M_{i}^{\text {snap }} \boldsymbol{\Sigma}_{i}
$$

where $\Lambda_{i}$ is the diagonal matrix with the eigenvalues on the diagonal, $\boldsymbol{\Sigma}_{i}$ is a square matrix with columns that are the corresponding eigenvectors. We define the functions along the edge $F \in \mathcal{E}_{i}$

$$
\psi_{j, F}=\left.\psi_{j, \mathcal{E}_{i}}\right|_{F},
$$


where $\psi_{j, \mathcal{E}_{i}}$ is the linear combination of snapshot vectors with the coefficients being the coordinates of the $j$ th eigenvector.

Further, if $F$ is a common face of $\Omega_{i}$ and $\Omega_{j}$, we need to apply to POD procedure in order to eliminate linearly dependent functions. After POD process, we get $L_{F}$ functions $\psi_{1, F}, \cdots, \psi_{L_{F}, F}$. Then for $F \in \mathcal{E}_{H}$

$$
M_{H}^{I_{b}}(F)=\operatorname{span}\left\{\psi_{j, F}: 0 \leq j \leq L_{F}\right\} \quad \text { and } \quad M_{H}^{I_{b}}:=\bigcup_{F \in \mathcal{E}_{H}} M_{H}^{I_{b}}(F) .
$$

3.3.3. The Mutiscale Space II- $a$. We define $\mathcal{E}_{i}$ as a single coarse face and $\omega_{i}$ as the union of two coarse elements, $\Omega_{1}$ and $\Omega_{2}$, that share this face $\mathcal{E}_{i}$, see Figure $3(\mathrm{~A})$. The snapshot space will consist of all fine-grid functions and thus the local spectral problem is solved directly on a fine grid (locally). In this case, we solve the following eigenvalue problem in a coarse neighborhood $\omega_{i}$ (see Figure 3). Let $X_{h}\left(\omega_{i}\right)$ be the conforming finite element space of continuous piece-wise

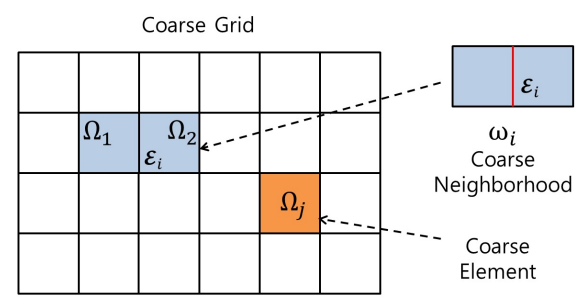

(A) Coarse grid for the space II-a

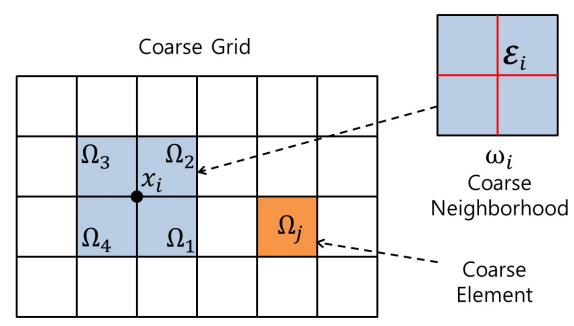

(в) Coarse grid for the space II-b

FigURE 3. Illustration of a coarse neighborhood and coarse element.

linear functions in $\mathcal{T}_{h}\left(\omega_{i}\right)$. For any given coarse neighborhood $\omega_{i}$ and the common face $\mathcal{E}_{i}$, we find $\lambda$ and $\psi \in X_{h}\left(\omega_{i}\right)$ such that

$$
\int_{\omega_{i}} \kappa(x) \nabla \psi \nabla z d x=\frac{\lambda}{H} \int_{\mathcal{E}_{i}} \bar{\kappa} \psi z d s, \quad \forall z \in X_{h}\left(\omega_{i}\right),
$$

with $\bar{\kappa}=2 \kappa_{1} \kappa_{2} /\left(\kappa_{1}+\kappa_{2}\right)$ and $\kappa_{1}=\left.\kappa\right|_{\Omega_{1}}$ and $\kappa_{2}=\left.\kappa\right|_{\Omega_{2}}$.

We order the eigenvalues so that $0 \leq \lambda_{i, 1} \leq \lambda_{i, 2} \leq \cdots \leq \lambda_{i, N_{i}}$, where $N_{i}$ is the number of vertices of $\mathcal{T}_{h}\left(\omega_{i}\right)$ and denote the corresponding eigenfunctions by $\psi_{i, 1}, \psi_{i, 2}, \ldots, \psi_{i, N_{i}}$, which form the space $V^{\text {off }}\left(\omega_{i}\right)$. Since the space $M_{H}$ is defined face-wise, we consider the traces of the constructed above eigenfunctions, denoted by $\psi_{l, F}, l=1, \cdots, N_{i}$, where $F$ is the common coarse face of $\omega_{i}$. These functions may be linearly dependent, so we choose linearly independent ones and further reduce their number by taking those that correspond to the smallest $L_{F}$ eigenvalues. These are the functions we use as a basis for the space $M_{H}$ :

$$
M_{H}^{I I_{a}}(F):=\operatorname{span}\left\{\psi_{j, F}, 1 \leq j \leq L_{F}\right\} \quad \text { so that } \quad M_{H}^{I I_{a}}:=\bigcup_{F \in \mathcal{E}_{H}} M_{H}^{I I_{a}}(F) .
$$


3.3.4. The Mutiscale Space II-b. For each node $\boldsymbol{x}_{i}$ we define $\mathcal{E}_{i}$ as the union of all coarse faces (edges) that share the node $\boldsymbol{x}_{i}$. Similarly, $\omega_{i}$ is the union of coarse elements that share a common node $\boldsymbol{x}_{i}$ (see Figure 3, where the coarse grid elements that form $\omega_{i}$ are $\Omega_{1}, \ldots, \Omega_{4}$ ).

We choose for the snapshot space the set of all fine-grid functions. Then the local spectral problem (14) is a finite element approximation of the following homogeneous Neumann eigenvalue problem: find $\psi^{\text {aux }} \in X_{h}\left(\omega_{i}\right)$ such that

$$
\int_{\omega_{i}} \kappa(x) \nabla \psi^{\mathrm{aux}} \nabla z d x=\lambda \int_{\omega_{i}} \tilde{\kappa} \psi^{\mathrm{aux}} z d s, \quad \forall z \in X_{h}\left(\omega_{i}\right) \quad \text { with } \quad \tilde{\kappa}:=\kappa \sum_{l=1}^{N_{v}}\left|\nabla \chi_{l}\right|^{2},
$$

where $\chi_{l}$ form partition of unity, e.g., piece-wise bilinear functions (see [22]).

Then, we order the eigenvalues as $0=\lambda_{i, 1} \leq \lambda_{i, 2} \leq \cdots$ and choose the $L_{i}$ eigenfunctions $\psi_{i, 1}^{\text {aux }}, \ldots, \psi_{i, L_{i}}^{\text {aux }}$, associated to the smallest eigenvalues and form basis $\psi_{i, j}=\chi_{i} \psi_{i, j}^{\text {aux }}$. Due to the properties of $\chi_{i}, \psi_{i, j} \in H_{0}^{1}(\Omega)$. Thus, we form $V^{\text {off }}\left(\omega_{i}\right)=\operatorname{span}\left\{\psi_{i, j}\right\}_{j=1}^{L_{i}}$.

Finally, we define

$$
M_{H}^{I I_{b}}(F)=\left.\bigcup_{F \in \mathcal{E}_{i}} V^{\mathrm{off}}\left(\omega_{i}\right)\right|_{F} \quad \text { and } \quad M_{H}^{I I_{b}}:=\bigcup_{F \in \mathcal{E}_{H}} M_{H}^{I I_{b}}(F),
$$

where $\left.V^{\text {off }}\left(\omega_{i}\right)\right|_{F}$ denotes the traces on $F$ of the functions in $V^{\text {off }}\left(\omega_{i}\right)$.

3.4. Solvability of the spectral mutiscale HDG method. Next, we discuss the stability and solvability of HDG method with multiscale spaces constructed in the previous section. To proceed, we need the following two assumptions on the local fine element spaces.

Assumption 3.2. For any $K \in \mathcal{T}_{h}, F^{*}$ an arbitrary edge of $K$, and $\mu \in M_{h}(F), F \in \partial K$, there exists a element $\boldsymbol{Z} \in \boldsymbol{V}(K)$ such that

$$
\begin{aligned}
& (\boldsymbol{Z}, \nabla w)=0, \quad \text { for all } w \in W(K), \\
& \left.\boldsymbol{Z} \cdot \boldsymbol{n}\right|_{F}=\mu, \quad \text { for all } F \in \partial K \backslash F^{*} \text {. }
\end{aligned}
$$

This assumption is trivially satisfied by all classical mixed finite elements as well as HDG elements (e.g. see $[12,13]$ for more details). The next assumption is for the stabilization parameter $\tau$.

Assumption 3.3. On each $F_{H} \in \mathcal{T}_{E}$, for any $T$ adjacent to $F_{H}$, i.e. $\bar{T} \cap F_{H} \neq \emptyset$, there exists at least one element $K \in \mathcal{T}_{T}$ adjacent to $F_{H}$, such that the stabilization operator $\tau>0$ on $F^{*}=F_{H} \cap \partial K$.

Now we state the solvability result of the method that is established by using approach developed in [13].

Theorem 3.4. If Assumptions 3.2 and 3.3 are satisfied, then for any $f$, the system (8) has unique solution.

We end this section by some discussions on the motivation of the construction of a coarse space $M_{H}$. In an ongoing research on the topic we have established some error estimates for the multiscale HDG method. Roughly speaking, for the primary unknown $u$, we have the bound:

$$
\left\|u-u_{h}\right\|_{\Omega} \leq C\left\|u-P_{M} u\right\|_{\frac{1}{2}, \partial \mathcal{T}_{H}}+C h^{\frac{1}{2}}\left\|u-P_{M} u\right\|_{0, \partial \mathcal{T}_{H}}+\mathcal{C}(h, \tau)
$$


Here $P_{M} u$ denotes the orthogonal $L^{2}$ projection onto the space $M_{H}$. The norms are defined as $\left\|u-P_{M} u\right\|_{s, \partial \mathcal{T}_{H}}:=\sum_{i=1}^{N}\left\|u-P_{M} u\right\|_{s, \partial \Omega_{i}}$. The last term, $\mathcal{C}(h, \tau)$, in this estimate involves several terms that depend on the mesh size $h$ and the stabilization parameter $\tau$ and converges to zero if the fine-grid size $h$ approaches to zero and $\tau=\mathcal{O}(1)$. As one can see, $\left\|u-P_{M} u\right\|_{\frac{1}{2}, \partial \mathcal{T}_{H}}$ is crucial in the estimate. With a further refined analysis, this term could be bounded by

$$
\left\|u-P_{M} u\right\|_{\frac{1}{2}, \partial \mathcal{T}_{H}} \leq \inf _{v \in M_{H}}\|u-v\|_{\frac{1}{2}, \partial \mathcal{T}_{H}}+\mathcal{C}(\tau, h),
$$

where $\mathcal{C}(h, \tau)$ has the same meaning as above. The above estimate is essential for choosing an appropriate space $M_{H}$ and a projection of the solution to $M_{H}$. From this estimate, we can see that one should construct the coarse space $M_{H}$ in such a way that it can provide a good approximation of $u$ in the $H^{\frac{1}{2}}$-norm along the coarse edges. In particular, we will need a majorizing norm which will define a local spectral problem and corresponding snapshot functions that will allow us to bound the error. This is a guideline for us in the above construction. Using (24) and (25), we can obtain error bounds similar to those in [19] for the MS Space II-b where the error scales as the inverse of the smallest eigenvalue that the corresponding eigenvector is not included in the coarse space. Because of the oversampling, we expect the approach for the MS Space II-b will have a better convergence rate compared to [17, 19].

\section{Numerical Results}

In this section we discuss representative numerical results. We compute the coarse-grid solution and study the error with respect to the reference solution (or the fine-grid solution of (4)). We note that the solution of (4) depends on both fine-scale and coarse-scale parameters, $h$ and $H$. We are mainly interested on the convergence (to the reference solution) when we add more spectral basis functions. We study the error behavior due to the addition of coarse basis functions for fixed value of $h$ and $H$.

We consider the domain $\Omega=(0,1)^{2}$ and divide $\Omega$ into $N=M \times M$ square coarse blocks, $\left\{\Omega_{i}\right\}_{i=1}^{N}$, which are unions of fine elements. In this case $H=1 / M$ is the coarse mesh parameter. Inside each subdomain $\Omega_{i}$, we generate a structured triangulation with $m$ subintervals in each coordinate direction (and thus $h=1 /(M m)$ is the fine mesh parameter). We consider the solution of Equation (4) with $f=1$ and the stabilization parameter $\tau=1$ on each edge (coarse and fine edge). We test our methods on two different distributions of the coefficient $\kappa(x)$ shown in Figure 4. $\kappa(x)=1$ are for the points in the white background and $\kappa(x)=\eta$ are for the points in the black regions. Example 1 represents the permeability of a media with highly permeable inclusions and relatively short isolated channels (not interconnected). Example 2 has one global channel. In our experiments, we consider $\eta$ to be $10^{4}$.

We consider the following finite element spaces which satisfy the assumptions of Subsection 3.4 in order to apply the mutiscale HDG method. $W_{h}$ and $\boldsymbol{V}_{h}$ consist of piece-wise linear, discontinuous functions on $\mathcal{T}_{h}, M_{h}$ of piece-wise linear, discontinuous functions in $\mathcal{E}_{h}$, and $M_{H}$ is the mutiscale finite element space, which is constructed in the previous section. To compute the error of $\boldsymbol{q}$, we define the weighted $L^{2}$-norm as following :

$$
\|\boldsymbol{q}\|_{\alpha, L^{2}(\Omega)}^{2}:=\int_{\Omega} \alpha|\boldsymbol{q}|^{2} d x .
$$




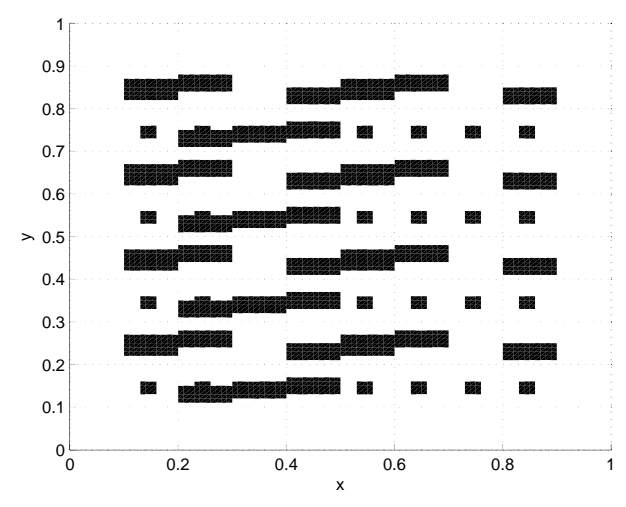

(A) Example 1

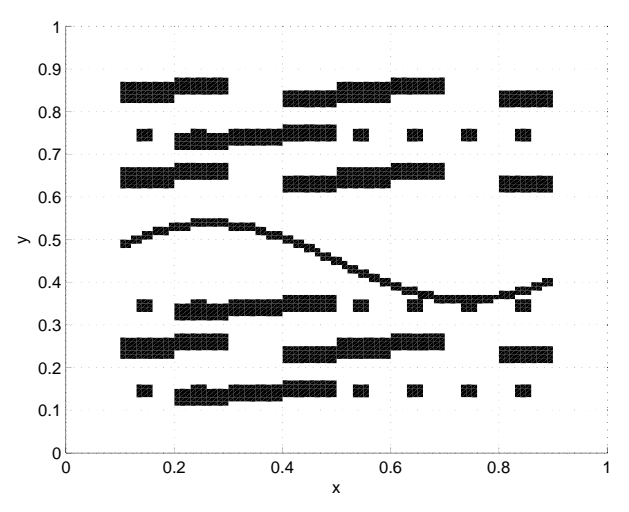

(B) Example 2

Figure 4. Topology of heterogeneous coefficient $\kappa$.

We compute the relative $L^{2}$-norm of the error for the solution $\left\|u_{h}^{*}-u_{H}\right\|_{L^{2}(\Omega)}$ and the relative weighted $L^{2}$-norm of the error for the velocity $\left\|\boldsymbol{q}_{h}^{*}-\boldsymbol{q}_{H}\right\|_{\alpha, L^{2}(\Omega)}$ - between the fine-scale solutions $\left(u_{h}^{*}, \boldsymbol{q}_{h}^{*}\right)$ and the coarse-grid solutions: (I) $\left(u_{H}^{I}, \boldsymbol{q}_{H}^{I}\right)$ introduced in the Subsection 3.3.1 and 3.3.2 or (II) $\left(u_{H}^{I I}, \boldsymbol{q}_{H}^{I I}\right)$ in Subsection 3.3.3 and 3.3.4.

4.1. Numerical results for Multiscale Spaces I applied to Example 1. In this subsection, we present the numerical experiments for the spaces introduced in Subsection 3.3.1 and 3.3 .2 .

TABLE 1. Numerical results for Example 1 when using Multiscale Spaces I (Subsection 3.3.1 and 3.3.2) with increasing dimension of the coarse space, $h=$ $1 / 100, H=1 / 10, \eta=10^{4}$. "Dim." stands for the dimension of the coarse space.

\begin{tabular}{ccc||ccc}
\hline \multicolumn{3}{c||}{ Multiscale Space I-a } & \multicolumn{3}{c}{ Mutiscale Space I-b } \\
\hline Dim. & $\left\|u_{h}^{*}-u_{H}\right\|_{L^{2}(\Omega)}$ & $\left\|\boldsymbol{q}_{h}^{*}-\boldsymbol{q}_{H}\right\|_{\alpha, L^{2}(\Omega)}$ & Dim. & $\left\|u_{h}^{*}-u_{H}\right\|_{L^{2}(\Omega)}$ & $\left\|\boldsymbol{q}_{h}^{*}-\boldsymbol{q}_{H}\right\|_{\alpha, L^{2}(\Omega)}$ \\
\hline 180 & 0.6412 & 0.8028 & 180 & 0.6412 & 0.8028 \\
469 & 0.1208 & 0.3547 & 514 & 0.0393 & 0.2321 \\
682 & 0.0362 & 0.2181 & 738 & 0.0312 & 0.1395 \\
888 & 0.0204 & 0.1659 & 848 & 0.0266 & 0.1247 \\
\hline
\end{tabular}

In these numerical experiments, we add multiscale basis functions and study the error decay as the coarse space dimension increases. The coefficient $\kappa(x)$ are depicted on Figure 4 . The results for the errors are presented in Table 1 for $h=1 / 100$ and $H=1 / 10$. We report the dimension of the coarse spaces (designated by "Dim."). We remind that for $n$-th row of the table, we add $n$ eigenfunctions from both sides of the edge and remove linearly dependent vectors. For this reason, the dimensions of the coarse space do not form a geometric progression.

The results show a convergence to the reference solution (fine-grid solution). We observe a fast error decay when adding more coarse basis functions. In particular, for $H=1 / 10$, we only have the dimensions of coarse spaces 682 and 738 to get the solution error $3.6 \%$ and $3.1 \%$ 
for the MS Space I-a and I-b, respectively. This error is computed with respect to the finegrid solution with the fine-grid mesh size $h=1 / 100$ and the dimension of fine-scale system is 60,000. We observe from this table that the MS Space I-b performs slightly better compared to the MS Space I-a.

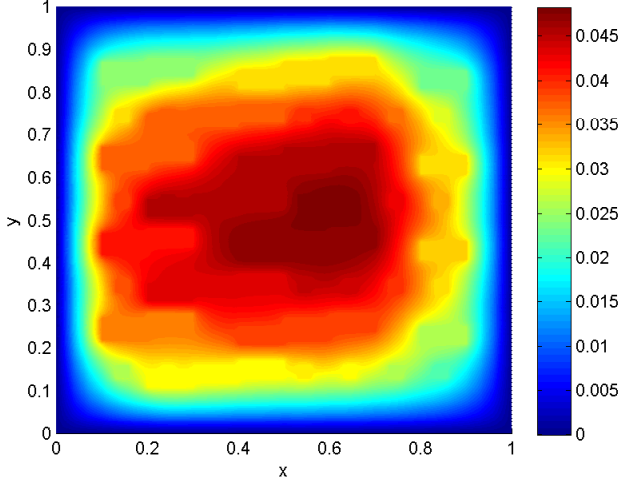

(A) Fine-scale solution

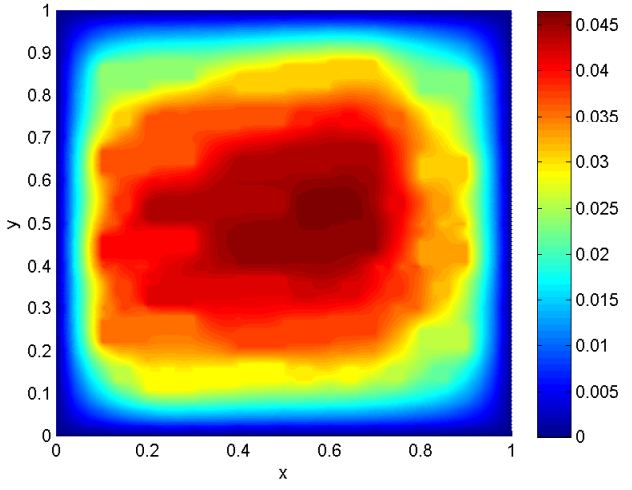

(C) Coarse-scale solution with Dim. $=720$

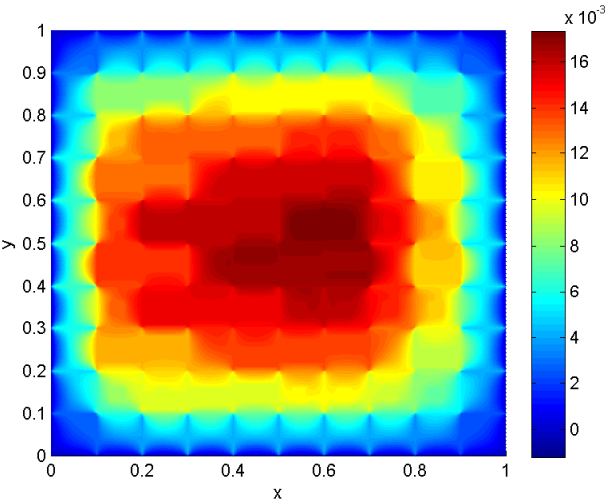

(B) Coarse-scale solution with Dim. $=180$

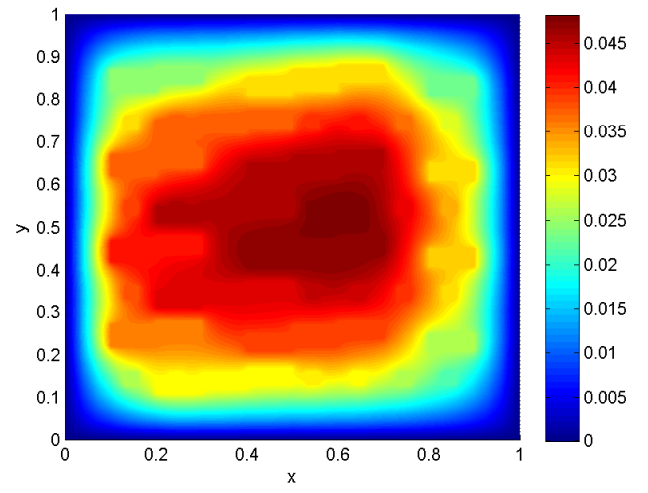

(D) Coarse-scale solution with Dim. $=1800$

Figure 5. Comparison of the coarse-scale solutions for MS Space II-a with the reference (fine-scale) solution.

4.2. Numerical results for Mutiscale Spaces II applied to Example 1. Next, we consider the spaces introduced in Section 3.3.3 and Section 3.3.4. We note that these spaces use an oversampling technique by solving local problems around faces and the numerical results corresponding to the MS Spaces II are better compared to MS Spaces I.

Figure 5 illustrates the effect of increasing the dimension of the coarse space in the MS Space II-a. We show the fine-scale solution and coarse-scale solutions computed with three different coarse space dimensions in Figure 5. We repeat the detailed numerical study described in Section 4.1. The results for the computation of errors with the MS Space II-a and II-b are presented in Table 2 and 3 for $h=1 / 100$ and $H=1 / 10$, respectively. When we use the coarse space with the dimension of 720 , we observe that the solution errors for the MS Space II-a 
TABLE 2. Numerical results for Multiscale Space II-a (Subsection 3.3.3) with increasing dimension of the coarse space, $h=1 / 100, H=1 / 10, \eta=10^{4}$. "Dim." stands for the dimension of the coarse space.

\begin{tabular}{ccc}
\hline Dim. & $\left\|u_{h}^{*}-u_{H}\right\|_{L^{2}(\Omega)}$ & $\left\|\boldsymbol{q}_{h}^{*}-\boldsymbol{q}_{H}\right\|_{\alpha, L^{2}(\Omega)}$ \\
\hline 180 & 0.6412 & 0.8028 \\
360 & 0.0561 & 0.2843 \\
540 & 0.0274 & 0.2306 \\
720 & 0.0251 & 0.1627 \\
\hline
\end{tabular}

TABLE 3. Numerical results for Multiscale Space II-b (Subsection 3.3.4) with increasing dimension of the coarse space, $h=1 / 100, H=1 / 10, \eta=10^{4}$. "Dim." stands for the dimension of the coarse space.

\begin{tabular}{ccc}
\hline Dim. & $\left\|u_{h}^{*}-u_{H}\right\|_{L^{2}(\Omega)}$ & $\left\|\boldsymbol{q}_{h}^{*}-\boldsymbol{q}_{H}\right\|_{\alpha, L^{2}(\Omega)}$ \\
\hline 360 & 0.1078 & 0.3290 \\
720 & 0.0384 & 0.0922 \\
1080 & 0.0381 & 0.0629 \\
\hline
\end{tabular}

and II-b are $2.5 \%$ and $3.8 \%$ and the velocity error are $16.3 \%$ and $9.2 \%$, respectively. Here, the dimension of the fine-grid system is 60,000 .

4.3. Numerical results for Example 2. Next, we consider a different permeability field for Example 2 (see Figure 4 ) with the contrast $\eta=10^{4}$. For this permeability field, a long channel in the middle of the domain is added to introduce a long-range effect in the solution. The numerical results are presented in Figure 6 and 7 where solution and velocity errors for these spaces are depicted. We note that the convergence behavior of these methods is similar to the previous permeability case with the errors that are slightly smaller.

We have also tested all the presented approaches for $\eta=10^{6}$ and observed similar accuracy. This shows that the method is robust with respect to the contrast.

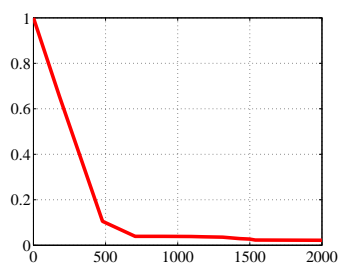

(A) MS space I-a

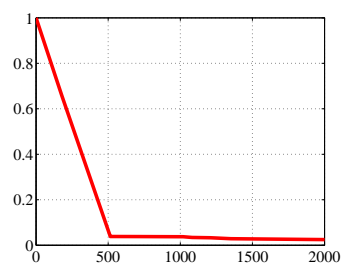

(B) MS space I-b

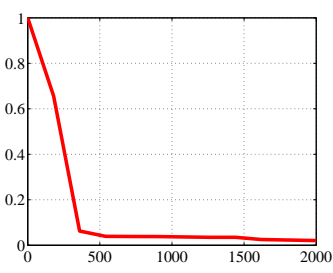

(c) MS space II-a

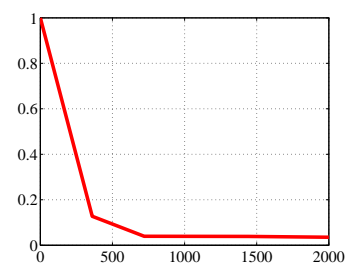

(D) MS space II-b

Figure 6. Numerical results for MS Spaces I and II applied to permeability field of Example 2 of Fig. 4 with $h=1 / 100, H=1 / 10$, and $\eta=10^{4}$ and increasing dimension of the coarse space. "Dim." stands for the dimension of the coarse space. The graphs show the relative error $\left\|u_{h}^{*}-u_{H}\right\|_{L^{2}(\Omega)}$ vs. the dimension of the coarse space. 


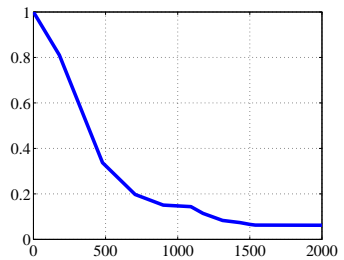

(A) MS space I-a

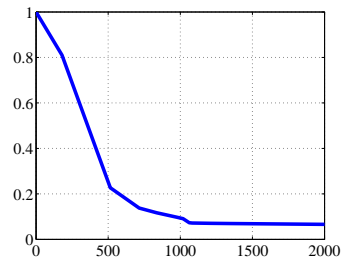

(B) MS space I-b

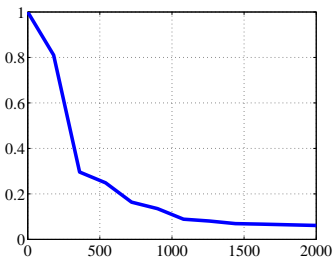

(C) MS space II-a

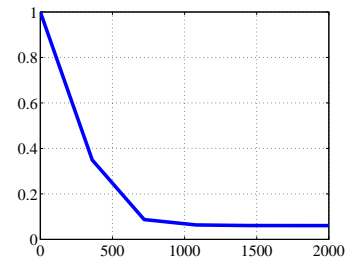

(D) MS space II-b

FIGURE 7. Numerical results for MS Spaces I and II applied to permeability field of Example 2 of Fig. 4 with $h=1 / 100, H=1 / 10$, and $\eta=10^{4}$ and increasing dimension of the coarse space. "Dim." stands for the dimension of the coarse space. The graphs show the relative error $\left\|\boldsymbol{q}_{h}^{*}-\boldsymbol{q}_{H}\right\|_{\alpha, L^{2}(\Omega)}$ vs. the dimension of the coarse space.

\section{Conclusion}

In this paper, we propose spectral mutiscale finite element methods for second order elliptic equations in the framework of the hybridizable discontinuous Galerkin finite element method. We propose several finite element spaces for the numerical traces. The main idea of the proposed method is to construct a low dimensional trace space $M_{H}$. In the paper, we present a general framework for defining trace spaces, which consists of three steps: (1) a partition of the coarse skeleton; (2) a construction of a local snapshot space; (3) a construction of the offline space and consequently a construction of the numerical trace space. Within this framework, we propose and test two classes of coarse spaces. The first class uses the boundaries of the coarse-grid subdomain to construct trace space, while the second class constructs the traces on the faces that are strictly within coarse blocks. In this regard, the second approach uses an oversampling technique where the information in larger domains are used in constructing multiscale basis functions. The presented numerical results for both methods show that one can achieve a good accuracy with a few degrees of freedom along each edge and the approaches that use oversampling provide better accuracy. In our future work, we plan to present detailed error analysis.

\section{Acknowledgements}

Y. Efendiev's work is partially supported by the DOE and NSF (DMS 0934837 and DMS 0811180) while R. Lazarov's research was supported in parts by NSF (DMS-1016525).

\section{REFERENCES}

[1] J. Aarnes. On the use of a mixed multiscale finite element method for greater flexibility and increased speed or improved accuracy in reservoir simulation. SIAM J. Multiscale Modeling and Simulation, 2:421-439, 2004.

[2] J. Aarnes and Y. Efendiev. Mixed multiscale finite element for stochastic porous media flows. SIAM J. Sci. Comput., 30 (5):2319-2339, 2008.

[3] T. Arbogast. Analysis of a two-scale, locally conservative subgrid upscaling for elliptic problems. SIAM J. Numer. Anal., 42(2):576-598 (electronic), 2004.

[4] T. Arbogast. Homogenization-based mixed multiscale finite elements for problems with anisotropy. Multiscale Model. Simul., 9(2):624-653, 2011. 
[5] T. Arbogast and K. Boyd. Subgrid upscaling and mixed multiscale finite elements. SIAM J. Numer. Anal., 44(3):1150-1171 (electronic), 2006.

[6] T. Arbogast, L. Cowsar, M. Wheeler, and I. Yotov. Mixed finite element methods on nonmatching multiblock grids. SIAM J. Numer. Anal., 37(4):1295-1315, 2000.

[7] T. Arbogast and M. Gomez. A discretization and multigrid solver for a Darcy-Stokes system of three dimensional vuggy porous media. Comput. Geosci., 13(2):331-343, 2009.

[8] T. Arbogast, G. Pencheva, M. Wheeler, and I. Yotov. A multiscale mortar mixed finite element method. Multiscale Model. Simul., 6(1):319-346, 2007.

[9] T. Arbogast and H. Xiao. A miltiscale mortar mixed space based homogenization for heterogeneous elliptic problems. SIAM J. Numerical Analysis, 51 (1)(1):377-399, 2013.

[10] C. Bernardi, Y. Maday, and A. Patera. Domain decomposition by the mortar element method. In H. Kaper, M. Garbey, and G. Pieper, editors, Asymptotic and Numerical Methods for Partial Differential Equations with Critical Parameters, volume 384 of NATO ASI Series, pages 269-286. Springer Netherlands, 1993.

[11] C.-C. Chu, I. G. Graham, and T.-Y. Hou. A new multiscale finite element method for high-contrast elliptic interface problems. Math. Comp., 79(272):1915-1955, 2010.

[12] B. Cockburn, J. Gopalakrishnan, and R. Lazarov. Unified hybridization of discontinuous Galerkin, mixed, and continuous Galerkin methods for second order elliptic problems. SIAM J. Numer. Analysis, 47(2):13191365, 2009.

[13] B. Cockburn, W. Qiu, and K. Shi. Conditions for superconvergence of HDG methods for second-order elliptic problems. Math. Comp., 81(279):1327-1353, 2012.

[14] W. E and B. Engquist. Heterogeneous multiscale methods. Comm. Math. Sci., 1(1):87-132, 2003.

[15] J. Eberhard and G. Wittum. A coarsening multigrid method for flow in heterogeneous porous media. In Multiscale methods in science and engineering, volume 44 of Lect. Notes Comput. Sci. Eng., pages 111-132. Springer, Berlin, 2005.

[16] Y. Efendiev, J. Galvis, and T. Y. Hou. Generalized multiscale finite element methods (gmsfem). Journal of Computational Physics, 251:116-135, 2013.

[17] Y. Efendiev, J. Galvis, R. Lazarov, M. Moon, and M. Sarkis. Generalized multiscale finite element method. Symmetric interior penalty coupling. J. Comput. Phys., 255:1-15, 2013.

[18] Y. Efendiev, J. Galvis, R. Lazarov, and J. Willems. Robust domain decomposition preconditioners for abstract symmetric positive definite bilinear forms. ESAIM Math. Model. Numer. Anal., 46(5):1175-1199, 2012.

[19] Y. Efendiev, J. Galvis, and X. Wu. Multiscale finite element methods for high-contrast problems using local spectral basis functions. Journal of Computational Physics, 230:937-955, 2011.

[20] Y. Efendiev and T. Hou. Multiscale Finite Element Methods: Theory and Applications, volume 4 of Surveys and Tutorials in the Applied Mathematical Sciences. Springer, New York, 2009.

[21] Y. Efendiev, T. Hou, and V. Ginting. Multiscale finite element methods for nonlinear problems and their applications. Comm. Math. Sci., 2:553-589, 2004.

[22] J. Galvis and Y. Efendiev. Domain decomposition preconditioners for multiscale flows in high contrast media. SIAM J. Multiscale Modeling and Simulation, 8:1461-1483, 2010.

[23] I. Graham, P. O. Lechner, and R. Scheichl. Domain decomposition for multiscale PDEs. Numerische Mathematik, 106(4):589-626, 2007.

[24] M. Hinze and S. Volkwein. Proper orthogonal decomposition surrogate models for nonlinear dynamical systems: error estimates and suboptimal control. In P. Benner, V. Mehrmann, and D. Sorensen, editors, Dimension Reduction of Large-Scale Systems, volume 45 of Lecture Notes in Computational Science and Engineering, pages 261-306. Springer Berlin Heidelberg, 2005.

[25] T. Hou and X. Wu. A multiscale finite element method for elliptic problems in composite materials and porous media. J. Comput. Phys., 134:169-189, 1997.

[26] T. Hughes, G. Feijoo, L. Mazzei, and J. Quincy. The variational multiscale method - a paradigm for computational mechanics. Comput. Methods Appl. Mech. Engrg., 166:3-24, 1998.

[27] O. Iliev, R. Lazarov, and J. Willems. Variational multiscale finite element method for flows in highly porous media. Multiscale Model. Simul., 9(4):1350-1372, 2011.

[28] P. Jenny, S. Lee, and H. Tchelepi. Multi-scale finite volume method for elliptic problems in subsurface flow simulation. J. Comput. Phys., 187:47-67, 2003. 
[29] G. Pencheva and I. Yotov. Balancing domain decomposition for mortar mixed finite element methods. Numerical Linear Algebra with Applications, 10(1-2):159-180, 2003.

[30] A. Toselli and O. Widlund. Domain decomposition methods - Algorithms and Theory, volume 34 of Computational Mathematics. Springer-Verlag, 2005.

[31] P. Vassilevski. Multilevel block-factrorization preconditioners. Matrix-based analysis and algorithms for solving finite element equations. Springer-Verlag, New York, 2008.

[32] P. Vassilevski. Coarse spaces by algebraic multigrid: multigrid convergence and upscaling error estimates. Adv. Adapt. Data Anal., 3(1-2):229-249, 2011.

[33] M. Wheeler, G. Xue, and I. Yotov. A multiscale mortar multipoint flux mixed finite element method. ESAIM Math. Model. Numer. Anal., 46(4):759-796, 2012.

Dept. Mathematics, Texas A\&M University, College Station, TX 77843, USA

E-mail address: efendiev@math.tamu.edu

Dept. Mathematics, Texas A\&M University, College Station, TX 77843, USA

E-mail address: lazarov@math.tamu.edu

Dept. Mathematics, Texas A\&M University, College Station, TX 77843, USA

E-mail address: mmoon@math.tamu.edu

Dept. Mathematics, Texas A\&M University, College Station, TX 77843, USA

E-mail address: shike@math.tamu.edu 